Article

\title{
Subadditive Pre-Image Variational Principle for Bundle Random Dynamical Systems
}

\author{
Xianfeng Ma *, Zhongyue Wang and Hailin Tan \\ Department of Mathematics, East China University of Science and Technology, Shanghai 200237, China; \\ Y30190200@mail.ecust.edu.cn (Z.W.); 10162587@mail.ecust.edu.cn (H.T.) \\ * Correspondence: xianfengma@ecust.edu.cn
}

Received: 1 January 2020; Accepted: 22 February 2020; Published: 27 February 2020

\begin{abstract}
A central role in the variational principle of the measure preserving transformations is played by the topological pressure. We introduce subadditive pre-image topological pressure and pre-image measure-theoretic entropy properly for the random bundle transformations on a class of measurable subsets. On the basis of these notions, we are able to complete the subadditive pre-image variational principle under relatively weak conditions for the bundle random dynamical systems.
\end{abstract}

Keywords: random dynamical systems; variational principle; subadditive pre-image topological pressure

\section{Introduction}

The topological pressure and variational principle play an important role in statistical mechanics, ergodic theory, and dynamical systems [1-8]. The classical topological pressure for a single potential was first presented by Ruelle [9] for expansive maps. Falconer [10] introduced the topological pressure for a subadditive sequence of potentials on mixing repellers. Cao [11] extended this notion to general compact dynamical systems. The topological pressure for nonadditive sequences of potentials has proved to be a valuable tool in the study of multifractal formalism in dimension theory, especially for non-conformal dynamical systems $[10,12,13]$. In random dynamical systems (RDS), the earlier work on topological pressure for a single potential was due to Ledrappier [14] and Bogenschütz [1]. Kifer [15] generalized this notion to general bundle RDS and set up the corresponding variational principle. Using the topological pressure for a single potential, Gundlach [16] derived the uniqueness of equilibrium states for a large class of functions, and recently Feng [17] studied the multifractal structure of disintegrations of Gibbs measures.

The pre-image structure of a map has been studied by many authors [18-27]. Cheng and Newhouse et al. [28] defined a new version of pre-image measure-theoretic and topological entropies and obtained a variational principle for entropy. Zeng [29] presented the notion of pre-image topological pressure $P_{\text {pre }}(T, f)$ for a single potential $f$ and established a pre-image variational principle as

$$
P_{\text {pre }}(T, f)=\sup \left\{h_{\text {pre }, \mu}(T)+\mu(f): \mu \in \mathcal{M}(T)\right\},
$$

where $h_{\text {pre, } \mu}(T)$ is the pre-image measure-theoretic entropy, $\mathcal{M}(T)$ is the set of $T$-invariant measures on the compact metric space $X$. Zhu [30,31] formulated the notions in the whole space $\Omega \times X$ analogous to those in the deterministic case, and deduced the variational principles for the random pre-image topological entropy and pre-image topological pressure as follows:

$$
\sup \left\{h_{\text {pre }, \mu}(\phi): \mu \in \mathcal{M}(\phi)\right\}=h_{\text {pre }}(\phi),
$$


and for $f \in \mathbf{L}^{1}(\Omega, \mathcal{C}(X))$,

$$
P_{\text {pre }}(\phi, f)=\sup \left\{h_{\text {pre }, \mu}(\phi)+\mu(f): \mu \in \mathcal{M}(\phi)\right\},
$$

where $\mathcal{M}(\phi)$ is the set of $\phi$-invariant measures on $\Omega \times X$.

The subadditive topological pressure has been proven to be essential to estimate the lower bound of the Hausdorff dimension of non-conformal repellers in the deterministic dynamical systems [32]. Thus, it should be asked if there exists a random version of pre-image thermodynamic formalism for subadditive sequences of potentials, which might have some potential applications in the study of multifractal analysis of non-conformal random dynamical systems.

In this paper, we present the notion of subadditive pre-image topological pressure $P_{\text {pre }}(T, \Phi)$ for continuous random dynamical systems. By restricting the pressure function being not $-\infty$, we set up the variational principle as

$$
P_{\text {pre }}(T, \Phi)=\sup \left\{h_{\text {pre }, \mu}^{(r)}(T)+\Phi^{*}(\mu): \mu \in \mathcal{M}_{\mathbf{P}}^{1}(\mathcal{E}, T) \text { and } \Phi^{*}(\mu)>-\infty\right\},
$$

where $\Phi^{*}(\mu)$ is the limit function of the subadditive potentials with respect to the invariant measure $\mu$. As for the case of $-\infty$, the subadditive pre-image topological pressure is tightly relative to the limit function $\Phi^{*}(\mu)$, which is also $-\infty$ for all invariant measure $\mu$. Our result generalizes Zhu's notion of entropy to the pressure on a class of measurable subsets for the general bundle RDS. In particular, if we consider the special case, i.e., $\Omega$ consists of only one point, $\mathcal{E}=\Omega \times X$, and the additive function sequence, then our result contains Zeng's deterministic pre-image variational principle. We adopt Ledrappier and Walters' method for decomposing the measure on each fiber instead of Cheng and Zeng's approach for using the upper semi-continuous decomposition of the space in the deterministic case. Our method generalizes Kifer's proof of the standard additive bundle random variational principle, which is a generalization of Misiurewicz's elegant proof for the (non-relative) variational principle [33].

This paper is organized as follows. In Section 2, we give the main needed definitions and results related to the pre-image measure-theoretic entropy for the bundle RDS. In Section 3, we introduce the notion of subadditive pre-image topological pressure and give the power rule for the pressure function. In Section 4, we state and prove the subadditive pre-image variational principle. Conclusions are presented in the final section.

\section{Pre-Image Measure-Theoretic Entropy}

$(\Omega, \mathcal{F}, \mathbf{P})$ is a probability space together with an invertible $\mathbf{P}$-preserving transformation $\vartheta$, where $\mathcal{F}$ is assumed to be complete, countably generated and separated points. $(X, d)$ is a compact metric space together with the Borel $\sigma$-algebra $\mathcal{B}$. A set $\mathcal{E} \subset \Omega \times X$ is measurable with respect to the product $\sigma$-algebra $\mathcal{F} \times \mathcal{B}$ and such that the fibers $\mathcal{E}_{\omega}=\{x \in X:(\omega, x) \in \mathcal{E}\}, \omega \in \Omega$, are compact. A continuous bundle random dynamical system (RDS) over $(\Omega, \mathcal{F}, \mathbf{P}, \vartheta)$ is generated by map $T_{\omega}: \mathcal{E}_{\rightarrow} \rightarrow \mathcal{E}_{\downarrow \rightarrow \text { with }}$ iterates $T_{\omega}^{n}=T_{\vartheta n-1} \cdots \cdots T_{\vartheta \omega} T_{\omega}, n \geq 1$, so that the map $(\omega, x) \rightarrow T_{\omega} x$ is measurable and the map $x \rightarrow T_{\omega} x$ is continuous for P-almost all (a.a) $\omega$. The map $\Theta: \mathcal{E} \rightarrow \mathcal{E}$ defined by $\Theta(\omega, x)=\left(\vartheta \omega, T_{\omega} x\right)$ is called the skew product transformation. For more details please refer to [34].

Denote $\mathcal{P}_{\mathbf{P}}(\mathcal{E})=\left\{\mu \in \mathcal{P}_{\mathbf{P}}(\Omega \times X): \mu(\mathcal{E})=1\right\}$, where $\mathcal{P}_{\mathbf{P}}(\Omega \times X)$ is the space of probability measures on $\Omega \times X$ having the marginal $\mathbf{P}$ on $\Omega$. Any $\mu \in \mathcal{P}_{\mathbf{P}}(\mathcal{E})$ on $\mathcal{E}$ can be disintegrated as $\mathrm{d} \mu(\omega, x)=\mathrm{d} \mu_{\omega}(x) \mathrm{d} \mathbf{P}(\omega)$ (see [35]), where $\mu_{\omega}$ are regular conditional probabilities with respect to the $\sigma$-algebra $\mathcal{F}_{\mathcal{E}}$ formed by all sets $(A \times X) \cap \mathcal{E}$ with $A \in \mathcal{F} . \mathcal{M}_{\mathbf{P}}^{1}(\mathcal{E}, T)$ denotes the set of $\Theta$-invariant measures $\mu \in \mathcal{P}_{\mathbf{P}}(\mathcal{E}) . \mu$ is $\Theta$-invariant if and only if the disintegrations $\mu_{\omega}$ of $\mu$ satisfy $T_{\omega} \mu_{\omega}=\mu_{\vartheta \omega}$ P-a.s. [36]. $\mathcal{Q}=\left\{\mathcal{Q}_{i}\right\}$ is a finite measurable partition of $\mathcal{E}$, and $\mathcal{Q}(\omega)=\left\{\mathcal{Q}_{i}(\omega)\right\}$, where $\left\{\mathcal{Q}_{i}(\omega)\right\}=\left\{x \in \mathcal{E}_{\omega}:(\omega, x) \in \mathcal{Q}_{i}\right\}$, is a partition of $\mathcal{E}_{\omega}$. For each $\omega \in \Omega$, denote $\mathcal{B}_{\omega}=$ $\left\{B \cap \mathcal{E}_{\omega}: B \in \mathcal{B}\right\}, \mathcal{B}_{\omega}^{-}=\bigcap_{n \geq 0}\left(T_{\omega}^{n}\right)^{-1} \mathcal{B}_{\vartheta^{n} \omega}$. Similarly, denote $(\mathcal{F} \times \mathcal{B})_{\mathcal{E}}=\{C \cap \mathcal{E}: C \in \mathcal{F} \times \mathcal{B}\}$, $(\mathcal{F} \times \mathcal{B})_{\mathcal{E}}^{-}=\bigcap_{n \geq 0} \Theta^{-n}(\mathcal{F} \times \mathcal{B})_{\mathcal{E}}$. 
For $\mu \in \mathcal{P}_{\mathbf{P}}(\mathcal{E})$, the conditional entropy of $\mathcal{Q}$ given the $\sigma$-algebra $\mathcal{F}_{\mathcal{E}} \vee(\mathcal{F} \times \mathcal{B})_{\mathcal{E}}^{-} \subset(\mathcal{F} \times \mathcal{B})_{\mathcal{E}}$ is defined as usual (See Kifer [37]) by

$$
\begin{aligned}
& H_{\mu}\left(\mathcal{Q} \mid \mathcal{F}_{\mathcal{E}} \vee(\mathcal{F} \times \mathcal{B})_{\mathcal{E}}^{-}\right) \\
= & -\int \sum_{i} \mu\left(\mathcal{Q}_{i} \mid \mathcal{F}_{\mathcal{E}} \vee(\mathcal{F} \times \mathcal{B})_{\mathcal{E}}^{-}\right) \log \mu\left(\mathcal{Q}_{i} \mid \mathcal{F}_{\mathcal{E}} \vee(\mathcal{F} \times \mathcal{B})_{\mathcal{E}}^{-}\right) d \mathbf{P},
\end{aligned}
$$

where $\mu\left(\mathcal{Q}_{i} \mid \mathcal{F}_{\mathcal{E}} \vee(\mathcal{F} \times \mathcal{B})_{\mathcal{E}}^{-}\right)$is the conditional probability of the set $\mathcal{Q}_{i}$ given $\mathcal{F}_{\mathcal{E}} \vee(\mathcal{F} \times \mathcal{B})_{\mathcal{E}}^{-}$.

The pre-image measure-theoretic (relativized) entropy $h_{\mathrm{pre}, \mu}^{(r)}(T)$ of the RDS $T$ with respect to $\mu \in \mathcal{M}_{\mathbf{P}}^{1}(\mathcal{E}, T)$ is defined by the formula

$$
h_{\mathrm{pre}, \mu}^{(r)}(T)=\sup _{\mathcal{Q}} h_{\mathrm{pre}, \mu}^{(r)}(T, \mathcal{Q})
$$

where

$$
h_{\text {pre }, \mu}^{(r)}(T, \mathcal{Q})=\lim _{n \rightarrow \infty} \frac{1}{n} H_{\mu}\left(\bigvee_{i=0}^{n-1} \Theta^{-i} \mathcal{Q} \mid \mathcal{F}_{\mathcal{E}} \vee(\mathcal{F} \times \mathcal{B})_{\mathcal{E}}^{-}\right)
$$

and the supremum is taken over all finite or countable measurable partitions $\mathcal{Q}=\left\{\mathcal{Q}_{i}\right\}$ of $\mathcal{E}$ with finite conditional entropy $H_{\mu}\left(\mathcal{Q} \mid \mathcal{F}_{\mathcal{E}}\right)<\infty$. It should be noted that the supremum in the Equation (2) can be taken only over partitions $\mathcal{Q}$ of $\mathcal{E}$ into sets $Q_{i}$ of the form $Q_{i}=\left(\Omega \times P_{i}\right) \cap \mathcal{E}$, where $\mathcal{P}=\left\{P_{i}\right\}$ is a partition of $X$ into measurable sets, so that $Q_{i}(\omega)=P_{i} \cap \mathcal{E}_{\omega}$ (See $[1,37,38]$ for detail). The existence of the limit (3) follows from the formula $\Theta^{-1}\left(\mathcal{F}_{\mathcal{E}} \vee(\mathcal{F} \times \mathcal{B})_{\mathcal{E}}^{-}\right) \subset \mathcal{F}_{\mathcal{E}} \vee(\mathcal{F} \times \mathcal{B})_{\mathcal{E}}^{-}$and the standard subadditive argument (cf. Kifer Theorem II.1.1 in [37]).

It should be noted that the pre-image measure-theoretic entropy defined here is on a class of measurable subsets $\mathcal{E}$ of $\Omega \times X$ instead of Zhu's on the whole space $\Omega \times X$. In [30], Zhu gave the fiberwise expression for this notion, which can be seen as a generalization of Kifer's [15]. For the above measurable subset $\mathcal{E}$, we gave a similar fiberwise expression in Proposition 1 in [39]. For the completeness, we give the proof as follows.

\section{Proposition 1.}

$$
h_{\mathrm{pre}, \mu}^{(r)}(T, \mathcal{Q})=\lim _{n \rightarrow \infty} \frac{1}{n} \int H_{\mu_{\omega}}\left(\bigvee_{i=0}^{n-1}\left(T_{\omega}^{i}\right)^{-1} \mathcal{Q}\left(\vartheta^{i} \omega\right) \mid \mathcal{B}_{\omega}^{-}\right) \mathrm{d} \mathbf{P}(\omega)
$$

Proof. Note that for any $f \in \mathbf{L}^{1}(\Omega \times X, \mu)$ and $\bigcup_{x \in F}\{\omega\} \times B_{\omega} \in \mathcal{F}_{\mathcal{E}} \vee(\mathcal{F} \times \mathcal{B})_{\mathcal{E}}^{-}$where $F \in \operatorname{Pr}_{\Omega} \mathcal{F}_{\mathcal{E}}$ and $B_{\omega} \in \mathcal{B}_{\omega}^{-}, \operatorname{Pr}_{\Omega} \mathcal{F}_{\mathcal{E}}$ is the projection of $\mathcal{F}_{\mathcal{E}}$ into $\Omega$, we have

$$
\begin{aligned}
& \int_{F} \int_{B_{\omega}} E\left(f \mid \mathcal{F}_{\mathcal{E}} \vee(\mathcal{F} \times \mathcal{B})_{\mathcal{E}}^{-}\right)(\omega, x) d \mu_{\omega}(x) d \mathbf{P}(\omega) \\
= & \int_{\cup_{x \in F}\{\omega\} \times B_{\omega}} f(\omega, x) d \mu(\omega, x) \\
= & \int_{F} \int_{B_{\omega}} f_{\omega}(x) d \mu_{\omega}(x) d \mathbf{P}(\omega) \\
= & \int_{F} \int_{B_{\omega}} E\left(f_{\omega} \mid \mathcal{B}_{\omega}^{-}\right) d \mu_{\omega}(x) d \mathbf{P}(\omega)
\end{aligned}
$$

where $f_{\omega}(x)=f(\omega, x)$. Therefore,

$$
E\left(f \mid \mathcal{F}_{\mathcal{E}} \vee(\mathcal{F} \times \mathcal{B})_{\mathcal{E}}^{-}\right)(\omega, x)=E\left(f_{\omega} \mid \mathcal{B}_{\omega}^{-}\right)(x) \quad \mu-\text { a.e. }
$$


Hence, for any finite measurable partition $\mathcal{Q}$ of $\mathcal{E}$, we have

$$
I_{\mu}\left(\mathcal{Q} \mid \mathcal{F}_{\mathcal{E}} \vee(\mathcal{F} \times \mathcal{B})_{\mathcal{E}}^{-}\right)(\omega, x)=I_{\mu_{\omega}}\left(\mathcal{Q}(\omega) \mid \mathcal{B}_{\omega}^{-}\right)(x) \quad \mu \text {-a.e. }
$$

where $I .(\cdot \mid \cdot)$ denotes the standard conditional information function. Thus,

$$
H_{\mu}\left(\mathcal{Q} \mid \mathcal{F}_{\mathcal{E}} \vee(\mathcal{F} \times \mathcal{B})_{\mathcal{E}}^{-}\right)=\int I_{\mu_{\omega}}\left(\mathcal{Q}(\omega) \mid \mathcal{B}_{\omega}^{-}\right) \mathrm{d} \mathbf{P}(\omega)
$$

Since $\left(\Theta^{-i} \mathcal{Q}\right)(\omega)=\left(T_{\omega}^{i}\right)^{-1} \mathcal{Q}\left(\vartheta^{i} \omega\right)$ for any $i \in \mathbb{N}$, then

$$
H_{\mu}\left(\bigvee_{i=0}^{n-1} \Theta^{-i} \mathcal{Q} \mid \mathcal{F}_{\mathcal{E}} \vee(\mathcal{F} \times \mathcal{B})_{\mathcal{E}}^{-}\right)=\int H_{\mu_{\omega}}\left(\bigvee_{i=0}^{n-1}\left(T_{\omega}^{i}\right)^{-1} \mathcal{Q}\left(\vartheta^{i} \omega\right) \mid \mathcal{B}_{\omega}^{-}\right) \mathrm{d} \mathbf{P}(\omega)
$$

Dividing by $n$ and letting $n \rightarrow \infty$, we obtain the Equation (4).

Furthermore, through a rigorous investigation, all results with respect to the pre-image measure-theoretic entropy hold in our case by using the methods in [30]. In the sequel, we will use these results directly and not give these proofs since it seems that there is no different from the arguments in [30] except changing the symbols and restricting to the measurable subset $\mathcal{E}$.

\section{Subadditive Pre-Image Topological Pressure}

Denote $X_{\mathcal{E}}=\left\{x \in X: \exists \omega \in \Omega\right.$ such that $\left.x \in \mathcal{E}_{\omega}\right\}$, for each $x \in X_{\mathcal{E}}$, we always assume that $\mathcal{E}(x)=\left\{(\omega, y): \omega \in \Omega, y \in T_{\omega}^{-1} x\right\}$ is measurable with respect to the product $\sigma$-algebra $\mathcal{F} \times \mathcal{B}$. For each $k \in \mathbb{N},\left(T_{\omega}^{k}\right)^{-1} x \neq \varnothing \mathbf{P}$-almost all (a.a) $\omega$, and denote $\mathcal{E}(x, k)=\left\{(\omega, y): \omega \in \Omega, y \in\left(T_{\omega}^{k}\right)^{-1} x\right\}$. By the continuity of RDS T and Theorem III.30 in [40], it is not hard to see that $\mathcal{E}(x, k)$ is also measurable. Since for each $k \in \mathbb{N},\left(T_{\omega}^{k}\right)^{-1} x$ is compact in $\mathcal{E}_{\omega}$, this means (See Chaper III in [40]) that the mapping $\omega: \rightarrow\left(T_{\omega}^{k}\right)^{-1} x$ is measurable with respect to the Borel $\sigma$-algebra induced by the Hausdorff topology on the space $\mathcal{K}(X)$ and that the distance function $d\left(z,\left(T_{\omega}^{k}\right)^{-1} x\right)$ is measurable in $\omega \in \Omega$ for each $z \in X$.

For each $n \in \mathbb{N}$, a family of metrics $d_{n}^{\omega}$ on $\mathcal{E}_{\omega}$ is defined as

$$
d_{n}^{\omega}(y, z)=\max _{0 \leq i<n}\left(d\left(T_{\omega}^{i} y, T_{\omega}^{i} z\right)\right), \quad y, z \in \mathcal{E}_{\omega}
$$

where $T_{\omega}^{0}$ is the identity map. It is not hard to see that, for each $k \in \mathbb{N}$ and $x \in X_{\mathcal{E}}$, the set $\mathcal{E}^{(2)}(x, k)=$ $\left\{(\omega, y, z): y, z \in\left(T_{\omega}^{k}\right)^{-1} x\right\}$ belongs to the product $\sigma$-algebra $\mathcal{F} \times \mathcal{B}^{2}$ (See Proposition III.13 in [40]). Since, for each $m \in \mathbb{N}, \epsilon>0$ and a real number $a$, the set $\left\{(\omega, y, z) \in \mathcal{E}^{(2)}(x, k): d\left(T_{\omega}^{m} y, T_{\omega}^{m} z\right) \leq a \epsilon\right\}$ is measurable with respect to this product $\sigma$-algebra, then $d_{n}^{\omega}(y, z)$ depends measurably on $(\omega, y, z) \in$ $\mathcal{E}^{(2)}(x, k)$.

For each $n \in \mathbb{N}$ and $\epsilon>0$, a set $F \subset \mathcal{E}_{\omega}$ is said to be $(\omega, n, \epsilon)$-separated if $y, z \in F, y \neq z$ implies $d_{n}^{\omega}(y, z)>\epsilon$. Similarly, for a compact subset $K \subset \mathcal{E}_{\omega}, F \subset K$ is called to be $(\omega, n, \epsilon)$-separated for $K$ if $y, z \in F, y \neq z$ implies $d_{n}^{\omega}(y, z)>\epsilon$.

Due to the compactness, there exists a small natural number $s_{n}(\omega, \epsilon)$ such that $\operatorname{card}(F) \leq$ $s_{n}(\omega, \epsilon)<\infty$ for every $(\omega, n, \epsilon)$-separated $F$. Moreover, there always exists a maximal $(\omega, n, \epsilon)$-separated set $F$ in the sense that, for every $y \in \mathcal{E}_{\omega}$ with $y \notin F$, the set $F \cup\{y\}$ is not $(\omega, n, \epsilon)$-separated anymore. In particular, this is also true for any compact subset $K$ in $\mathcal{E}_{\omega}$, and we let $s_{n}(\omega, \epsilon, K)$ be the small natural number such that $\operatorname{card}(F) \leq s_{n}(\omega, \epsilon, K)<\infty$ for every $(\omega, n, \epsilon)$-separated set $F$ of $K$.

For each measurable in $(\omega, x)$ and continuous in $x \in \mathcal{E}_{\omega}$ function $f$ on $\mathcal{E}$, let

$$
\|f\|=\int\|f(\omega)\|_{\infty} \mathrm{d} \mathbf{P}, \quad \text { where } \quad\|f(\omega)\|_{\infty}=\sup _{x \in \mathcal{E}_{\omega}}|f(\omega, x)| .
$$


and $\mathbf{L}_{\mathcal{E}}^{1}(\Omega, \mathcal{C}(X))$ be the space of such functions $f$ with $\|f\|<\infty$ and identify $f$ and $g$ provided $\|f-g\|=0$, then $\mathbf{L}_{\mathcal{E}}^{1}(\Omega, \mathcal{C}(X))$ is a Banach space with the norm $\|\cdot\|$.

Let $\Phi=\left\{f_{n}\right\}_{n=1}^{\infty}$ be a sequence functions on $\mathcal{E}$ such that each $f_{n}$ is measurable in $\omega$ and continuous in $x$ on $\mathcal{E}$. These functions are measurable in $(\omega, x)$ in view of Lemma III.14 from [37]. $\Phi$ is called subadditive if for any $(\omega, x) \in \mathcal{E}$ and $m, n \in \mathbb{N}$,

$$
f_{n+m}(\omega, x) \leq f_{n}(\omega, x)+f_{m}\left(\Theta^{n}(\omega, x)\right) .
$$

If $f_{1} \in \mathbf{L}_{\mathcal{E}}^{1}(\Omega, \mathcal{C}(X))$ and this inequality holds, then a simple calculation indicates that each $f_{n} \in$ $\mathbf{L}_{\mathcal{E}}^{1}(\Omega, \mathcal{C}(X))$. In the following, we always assume $\Phi$ satisfying these conditions.

For any $\Theta$-invariant measure $\mu$, denote

$$
\Phi^{*}(\mu)=\lim _{n \rightarrow \infty} \frac{1}{n} \int f_{n} \mathrm{~d} \mu .
$$

Existence of the limit follows from the well-known subadditive argument. If we denote $\Phi^{k}=\left\{f_{k n}\right\}_{n=1}^{\infty}$ for any $k \in \mathbb{N}$, then $\left(\Phi^{k}\right)^{*}(\mu)=k \Phi^{*}(\mu)$. Most of the above mentioned in this section can be found in references [34,39]. We list them here for completeness.

For $\Phi=\left\{f_{n}\right\}_{n=1}^{\infty}$ assumed as above, $k, n \in \mathbb{N}, k \geq n, \epsilon>0, x \in X_{\mathcal{E}}$ with $\left(T_{\omega}^{k}\right)^{-1} x \neq \varnothing$, and an $(\omega, n, \epsilon)$-separated set $E$ of $\left(T_{\omega}^{k}\right)^{-1} x$ such that $E \subset\left(T_{\omega}^{k}\right)^{-1} x \subset \mathcal{E}_{\omega}$, denote

$$
P_{\text {pre }, n, \omega}\left(T, \Phi, \epsilon,\left(T_{\omega}^{k}\right)^{-1} x\right)=\sup _{E} \sum_{y \in E} \exp f_{n}(\omega, y)
$$

where $E$ is taken over all $(\omega, n, \epsilon)$-separated sets of $\left(T_{\omega}^{k}\right)^{-1} x$ in $\mathcal{E}_{\omega}$. For $\left(T_{\omega}^{k}\right)^{-1} x=\varnothing$, we set $P_{\text {pre, } n, \omega}\left(T, f, \epsilon,\left(T_{\omega}^{k}\right)^{-1} x\right)=0$ for all $n$ and $\epsilon$, and then the function $P_{\text {pre }, n, \omega}\left(T, f, \epsilon,\left(T_{\omega}^{k}\right)^{-1} x\right)$ is well-defined. In the sequel, we always assume that, for each $k \in \mathbb{N}$ and $x \in X_{\mathcal{E}},\left(T_{\omega}^{k}\right)^{-1} x \neq \varnothing$. Alternatively, we can assume that the map $T_{\omega}$ is a surjection for each $\omega$. Clearly, the above supremum can be taken only over all maximal $(\omega, n, \epsilon)$-separated sets.

The following auxiliary result, which relies on Kifer's work [15] restricted on compact subset and nonrandom positive number $\epsilon$, provides basic measurability properties needed in what follows. We prove the additive case in Lemma 2 in [39]. By replacing $S_{n} f$ by $f_{n}$ and $f$ by $\Phi$, respectively, we make a little change and give a similar argument for the subadditive case as follows.

Lemma 1. Suppose that $\mathcal{E}(x)=\left\{(\omega, y): \omega \in \Omega, y \in T_{\omega}^{-1} x\right\}$ is measurable for each $x \in X_{\mathcal{E}}$ as above, then, for any $k, n \in \mathbb{N}, k \geq n$ and a nonrandom small positive number $\epsilon$, the function $P_{\mathrm{pre}, n, \omega}\left(T, \Phi, \epsilon,\left(T_{\omega}^{k}\right)^{-1} x\right)$ is measurable in $\omega$, and for any $\delta>0$, there exists a family of maximal $(\omega, n, \epsilon)$-separated sets $G_{\omega} \subset\left(T_{\omega}^{k}\right)^{-1} x \subset$ $\mathcal{E}_{\omega}$ satisfying

$$
\sum_{y \in G_{\omega}} \exp f_{n}(\omega, y) \geq(1-\delta) P_{\text {pre }, n, \omega}\left(T, \Phi, \epsilon,\left(T_{\omega}^{k}\right)^{-1} x\right)
$$

and depending measurably on $\omega$ in the sense that $G=\left\{(\omega, x): x \in G_{\omega}\right\} \in \mathcal{F} \times \mathcal{B}$, which also means that the mapping $\omega \rightarrow G_{\omega}$ is measurable with respect to the Borel $\sigma$-algebra induced by the Hausdorff topology on the $\mathcal{K}(X)$ of compact subsets of $X$. In particular, the supremum in the definition of $P_{\text {pre, } n, \omega}\left(T, \Phi, \epsilon,\left(T_{\omega}^{k}\right)^{-1} x\right)$ can be taken only over measurable in $\omega$ families of $(\omega, n, \epsilon)$-separated sets. 
Proof. Fix $x \in X_{\mathcal{E}}$. For $q, n \in \mathbb{N}_{+}$set

$$
\begin{aligned}
D_{q} & =\left\{\left(\omega, x_{1}, \ldots, x_{q}\right): \omega \in \omega, x_{i} \in\left(T_{\omega}^{k}\right)^{-1} x, \forall i\right\}, \\
E_{q}^{n} & =\left\{\left(\omega, x_{1}, \ldots, x_{q}\right) \in D_{q}: d_{n}^{\omega}\left(x_{i}, x_{j}\right)>\epsilon, \forall i \neq j\right\}, \\
E_{q}^{n, l} & =\left\{\left(\omega, x_{1}, \ldots, x_{q}\right) \in D_{q}: d_{n}^{\omega}\left(x_{i}, x_{j}\right) \geq \epsilon+1 / l, \forall i \neq j\right\}, \\
E_{q}^{n}(\omega) & =\left\{\left(x_{1}, \ldots, x_{q}\right):\left(\omega, x_{1}, \ldots, x_{q}\right) \in E_{q}^{n}\right\} \\
E_{q}^{n, l}(\omega) & =\left\{\left(x_{1}, \ldots, x_{q}\right):\left(\omega, x_{1}, \ldots, x_{q}\right) \in E_{q}^{n, l}\right\}
\end{aligned}
$$

Observe that $D_{q} \in \mathcal{F} \times \mathcal{B}^{q}$, where $\mathcal{B}^{q}$ is the product $\sigma$-algebra on the product of $q$ copies of $X$. This follows from Theorem III.30 in [40] since

$$
d_{q}\left(\left(x_{1}, \ldots, x_{q}\right),\left(y_{1}, \ldots, y_{q}\right)\right)=\sum_{i=1}^{q} d\left(x_{i}, y_{i}\right)
$$

is the distance function on $X^{q}$; and, if $\mathcal{E}^{(q)}(x, k)$ denotes the product of $q$ copies of $\mathcal{E}(x, k)$, then

$$
d_{q}\left(\left(x_{1}, \ldots, x_{q}\right), \mathcal{E}^{(q)}(x, k)\right)=\sum_{i=1}^{q} d\left(x_{i}, \mathcal{E}(x, k)\right)
$$

is measurable in $\omega$ for each $\left(x_{1}, \ldots, x_{q}\right) \in X^{q}$. Next, define $q(q-1) / 2$ measurable functions $\psi_{i j}, 1 \leq$ $i<j \leq q$, on $D_{q}$ by $\psi_{i j}\left(\omega, x_{1}, \ldots, x_{q}\right)=d_{n}^{\omega}\left(x_{i}, x_{j}\right)$. Then,

$$
E_{q}^{n, l}=\bigcup_{1 \leq i<j \leq q} \psi_{i j}^{-1}[\epsilon+1 / l, \infty) \in \mathcal{F} \times \mathcal{B}^{q}
$$

By the continuity of the RDS $T$, each $E_{q}^{n, l}(\omega)$ is a closed subset of $\mathcal{E}^{(q)}(x, k)$, and so it is compact. Clearly, $E_{q}^{n, l} \uparrow E_{q}^{n}$ and $E_{q}^{n, l}(\omega) \uparrow E_{q}^{n}(\omega)$ as $l \rightarrow \infty$, in particular, $E_{q}^{n} \in \mathcal{F} \times \mathcal{B}^{q}$.

Let $s_{n}(\omega, \epsilon)$ be the largest cardinality of all $(\omega, n, \epsilon)$-separated set in $\mathcal{E}(x, k)$ and set $t_{n, l}(\omega, \epsilon)=$ $\max \left\{q: E_{q}^{n, l}(\omega) \neq \varnothing\right\}$. By Theorem III.23 in [40], it follows that

$$
\left\{\omega: t_{n, l}(\omega, \epsilon) \geq q\right\}=\left\{\omega: E_{q}^{n, l}(\omega) \neq \varnothing\right\}=\operatorname{Pr}_{\Omega} E_{q}^{n, l} \in \mathcal{F},
$$

where $\operatorname{Pr}_{\Omega}$ is the projection of $\Omega \times X^{q}$ to $\Omega$, and so $t_{n, l}(\omega, \epsilon)$ is measurable in $\omega$. Now,

$$
\left\{\omega: s_{n}(\omega, \epsilon) \geq q\right\}=\left\{\omega: E_{q}^{n}(\omega) \neq \varnothing\right\}=\bigcup_{m=1}^{\infty} \bigcap_{k=m}^{\infty}\left\{\omega: E_{q}^{n, l}(\omega) \neq \varnothing\right\} \in \mathcal{F},
$$

and so $s_{n}(\omega, \epsilon)$ is measurable in $\omega$ as well. Since $s_{n}(\omega, \epsilon) \geq t_{n, l}(\omega, \epsilon)$ for all $l \geq 1$, it follows that $t_{n, l}(\omega, \epsilon) \uparrow s_{n}(\omega, \epsilon)$ as $l \rightarrow \infty$, and so $t_{n, l}(\omega, \epsilon)=s_{n}(\omega, \epsilon)$ for all $k$ large enough (depending on $n$ and $\omega)$. By Lemma III.39 in [40], each function

$$
g_{q, l}=\sup \left\{\sum_{i=1}^{q} \exp f_{n}\left(\omega, x_{i}\right):\left(x_{1}, \ldots, x_{q}\right) \in E_{q}^{n, l}(\omega)\right\}
$$

is measurable, and so both functions $g_{q}=\sup _{l \geq 1} g_{q, l}$ and $P_{\text {pre, } n, \omega}\left(T, \Phi, \epsilon,\left(T_{\omega}^{k}\right)^{-1} x\right)=$ $\max _{1 \leq q \leq s_{n}(\omega, \epsilon)} g_{q}(\omega)$ are measurable.

For each constant $\delta>0$, the set

$$
F_{q, \delta}^{n}=\left\{\left(\omega, x_{1}, \ldots, x_{q}\right) \in D_{q}: \sum_{i=1}^{q} \exp f_{n}\left(\omega, x_{i}\right) \geq(1-\delta) g_{q}(\omega)\right\}
$$


belongs to $\mathcal{F} \times \mathcal{B}^{q}$. Hence,

$$
G_{q, \delta}^{n}=F_{q, \delta}^{n} \cap E_{q}^{n} \in \mathcal{F} \times \mathcal{B}^{q} \quad \text { and } \quad G_{q, \delta}^{n, l}=F_{q, \delta}^{n} \cap E_{q}^{n, l} \in \mathcal{F} \times \mathcal{B}^{q} .
$$

Set

$$
\begin{aligned}
& G_{q, \delta}^{n}(\omega)=\left\{\left(x_{1}, \ldots, x_{q}\right):\left(\omega, x_{1}, \ldots, x_{q}\right) \in G_{q, \delta}^{n}\right\}, \\
& G_{q, \delta}^{n, l}(\omega)=\left\{\left(x_{1}, \ldots, x_{q}\right):\left(\omega, x_{1}, \ldots, x_{q}\right) \in G_{q, \delta}^{n, l}\right\} .
\end{aligned}
$$

Obverse that $G_{q, \delta}^{n, l}(\omega)$ are compact sets and $G_{q, \delta}^{n, l}(\omega) \uparrow G_{q, \delta}^{n}(\omega)$ as $l \rightarrow \infty$. The sets

$$
\widetilde{\Omega}_{q, l}=\left\{\omega: t_{n, l}(\omega, \epsilon)=s_{n}(\omega, \epsilon)=q\right\} \cap\left\{\omega: G_{q, \delta}^{n, l}(\omega) \neq \varnothing\right\}
$$

are, clearly, measurable, and the sets $\Omega_{q, l}=\widetilde{\Omega}_{q, l} \backslash \widetilde{\Omega}_{q, l-1}, l=1,2, \ldots$, with $\widetilde{\Omega}_{q, 0}=\varnothing$ are measurable, disjoint and $\bigcup_{q, l \geq 1} \Omega_{q, l}=\Omega$. Thus (see Theorem III.30 in [40]), the multifunction $\Psi_{q, l, \delta}$ defined by $\Psi_{q, l, \delta}(\omega)=G_{q, \delta}^{n, l}(\omega)$ for $\omega \in \Omega_{q, l}$ is measurable, and it admits a measurable selection $\sigma_{q, l, \delta}$ which is measurable map $\sigma_{q, l, \delta}: \Omega_{q, l} \rightarrow X^{q}$ such that $\sigma_{q, l, \delta}(\omega) \in G_{q, \delta}^{n, l}(\omega)$ for all $\omega \in \Omega_{q, l}$. Let $\zeta_{q}$ be the multifunction form $X^{q}$ to $q$-point subsets of $X$ defined by $\zeta_{q}\left(x_{1}, \ldots, x_{q}\right)=\left\{x_{1}, \ldots, x_{q}\right\} \subset X$. Then, $\zeta_{q} \circ \sigma_{q, l, \delta}$ is a multifunction assigning to each $\omega \in \Omega_{q, l}$ a maximal $(\omega, n, \epsilon)$-separated set $G_{\omega} \subset\left(T_{\omega}^{k}\right)^{-1} x$ in $\mathcal{E}_{\omega}$ for which the Equation (5) holds true.

For any open set, $U \subset X$, denote $V_{U}^{q}(i)=\left\{\left(x_{1}, \ldots, x_{q}\right) \in X^{q}: x_{i} \in U\right\}$ which is an open set of $X^{q}$. Then, clearly,

$$
\left\{\omega \in \Omega_{q, l}: \zeta_{q} \circ \sigma_{q, l, \delta}(\omega) \cap U \neq \varnothing\right\}=\bigcup_{i=1}^{q} \sigma_{q, l, \delta}^{-1} V_{U}^{q}(i) \in \mathcal{F} .
$$

Define the random variable $m_{q}$ by $m_{q}(\omega)=l$ for all $\omega \in \Omega_{q, l}$ and set $\Lambda_{\delta}(\omega)=\zeta_{s_{n}(\omega, \epsilon)} \circ$ $\sigma_{s_{n}}(\omega, \epsilon), m_{q}(\omega), \delta(\omega) ;$ then,

$$
\left\{\omega: \Lambda_{\delta}(\omega) \cap U \neq \varnothing\right\}=\bigcup_{q, l=1}^{\infty}\left\{\omega \in \Omega_{q, l}: \zeta_{q} \circ \sigma_{q, l, \delta}(\omega) \cap U \neq \varnothing\right\} \in \mathcal{F}
$$

Hence, $\Lambda_{\delta}$ is measurable multifunction which assigns to each $\omega \in \Omega$ a maximal $(\omega, n, \epsilon)$-separated set $G_{\omega}$ for which Equation (5) holds true and Lemma 1 follows since $\delta>0$ is arbitrary.

For the function sequence $\Phi$ assumed as above and any positive number $\epsilon, n, k \in \mathbb{N}$, if $\sup _{x \in X_{\mathcal{E}}} P_{\text {pre }, n, \omega}\left(T, \Phi, \epsilon,\left(T_{\omega}^{k}\right)^{-1} x\right) \in \mathbf{L}_{\mathcal{E}}^{1}(\Omega, \mathcal{C}(X))$, then we can set

$$
P_{\text {pre }}(T, \Phi, \epsilon)=\limsup _{n \rightarrow \infty} \frac{1}{n} \sup _{k \geq n} \int \log \sup _{x \in X_{\mathcal{E}}} P_{\text {pre }, n, \omega}\left(T, \Phi, \epsilon,\left(T_{\omega}^{k}\right)^{-1} x\right) d \mathbf{P}(\omega)
$$

It should be noted that, though we set $x \in X_{\mathcal{E}}$, in fact, only those points such that $x \in \mathcal{E}_{\vartheta^{k} \omega}$ acts in the function $P_{\text {pre }}(T, \Phi, \epsilon)$.

The subadditive pre-image (relativized) topological pressure of the function sequence $\Phi$ for the bundle RDS $T$ is defined as

$$
P_{\text {pre }}(T, \Phi)=\lim _{\epsilon \rightarrow 0} P_{\text {pre }}(T, \Phi, \epsilon)
$$

and the limit exists since $P_{\text {pre }}(T, \Phi, \epsilon)$ is monotone in $\epsilon$ and, in fact, $\lim _{\epsilon \rightarrow 0}$ equal sup $\operatorname{su}_{\epsilon \rightarrow 0}$. 
Remark 1. If we let $f=0$ and consider the whole product space $\Omega \times X$ in this definition, then it is the pre-image topological entropy for the bundle RDS T defined by Zhu [30] through the separated set:

$$
h_{\text {pre }}^{(r)}(T)=\lim _{\epsilon \rightarrow 0} \limsup _{n \rightarrow \infty} \frac{1}{n} \sup _{k \geq n} \int \log \sup _{x \in X} s_{n}\left(\omega, \epsilon,\left(T_{\omega}^{k}\right)^{-1} x\right) \mathrm{d} \mathbf{P}(\omega)
$$

where $s_{n}\left(\omega, \epsilon,\left(T_{\omega}^{k}\right)^{-1} x\right)$ is the largest cardinality of an $(\omega, n, \epsilon)$-separated set of $\left(T_{\omega}^{k}\right)^{-1} x$.

Remark 2. If the measure space $\Omega$ consists of the single point, i.e., $\Omega=\{\omega\}$, and the function sequence is the trivial one, namely, $f_{n}=f$, for each $n$, which is the special additive case for the subadditive function sequence, then it is not difficult to see that the above definition is just the pre-image topological pressure defined by Zeng [29] in the deterministic dynamical systems.

For a given $m \in \mathbb{N}_{+}$, if we replace $\vartheta$ by $\vartheta^{m}$ and consider the bundle RDS $T^{m}$ defined by $\left(T^{m}\right)_{\omega}^{n}=$ $T_{\vartheta(n-1) m}^{m} \cdots T_{\vartheta^{m}}^{m} T_{\omega}^{m}$, i.e. $\left(T^{m}\right)_{\omega}^{n}=T_{\omega}^{m n}$, then the subadditive pre-image topological pressure has the following power rule.

Lemma 2. Let $T$ be a continuous bundle $R D S$ on $\mathcal{E}$ and $\Phi$ be a subadditive sequence of potentials. Then, for any $m>0, P_{\text {pre }}\left(T^{m}, \Phi^{m}\right)=m P_{\text {pre }}(T, \Phi)$.

Proof. Let $n \in \mathbb{N}, k \geq n$ and $x \in X_{\mathcal{E}}$. If $E$ is an $(\omega, n, \epsilon)$-separated set of $\left(\left(T^{m}\right)_{\omega}^{k}\right)^{-1} x$ for $T^{m}$, then $E$ is also an $(\omega, m n, \epsilon)$-separated set of $\left(T_{\omega}^{m k}\right)^{-1} x$ for $T$. Since $\left(T^{m}\right)_{\omega}^{n}=T_{\omega}^{m n}$,

$$
P_{\text {pre }, n, \omega}\left(T^{m}, \Phi^{m}, \epsilon,\left(\left(T^{m}\right)_{\omega}^{k}\right)^{-1} x\right) \leq P_{\text {pre }, m n, \omega}\left(T, \Phi, \epsilon,\left(T_{\omega}^{m k}\right)^{-1} x\right)
$$

Hence,

$$
\begin{aligned}
& P_{\text {pre }}\left(T^{m}, \Phi^{m}, \epsilon\right) \\
= & \limsup _{n \rightarrow \infty} \frac{1}{n} \sup _{k \geq n} \int \log \sup _{x \in X_{\mathcal{E}}} P_{\text {pre }, n, \omega}\left(T^{m}, \Phi^{m}, \epsilon,\left(\left(T^{m}\right)_{\omega}^{k}\right)^{-1} x\right) d \mathbf{P}(\omega) \\
\leq & \limsup _{n \rightarrow \infty} \frac{1}{n} \sup _{k \geq n} \int \log \sup _{x \in X_{\mathcal{E}}} P_{\text {pre }, m n, \omega}\left(T, \Phi, \epsilon,\left(T_{\omega}^{m k}\right)^{-1} x\right) d \mathbf{P}(\omega) \\
\leq & m \limsup _{n \rightarrow \infty} \frac{1}{m n} \sup _{k \geq m n} \int \log \sup _{x \in X_{\mathcal{E}}} P_{\text {pre }, m n, \omega}\left(T, \Phi, \epsilon,\left(T_{\omega}^{k}\right)^{-1} x\right) d \mathbf{P}(\omega) \\
\leq & m \limsup _{n \rightarrow \infty} \frac{1}{n} \sup _{k \geq n} \int \log \sup _{x \in X_{\mathcal{E}}} P_{\text {pre }, n, \omega}\left(T, \Phi, \epsilon,\left(T_{\omega}^{k}\right)^{-1} x\right) d \mathbf{P}(\omega) \\
\leq & m P_{\text {pre }}(T, \Phi, \epsilon) .
\end{aligned}
$$

Therefore, $P_{\text {pre }}\left(T^{m}, \Phi^{m}\right) \leq m P_{\text {pre }}(T, \Phi)$.

Since $T^{m}$ is continuous, then for any $\epsilon>0$, some small $\delta>0$ exists such that $d(y, z)<\delta$ with $y, z \in \mathcal{E}_{\omega}$ implies $d_{\omega}^{m}(y, z)<\epsilon$. For any positive integer $n$, one can find some integer $l$ such that $m n \leq l<m(n+1)$. Notice that any $(\omega, l, \epsilon)$-separated set for $T$ is also $(\omega, n, \delta)$-separate for 
$T^{m}$. By the subadditivity of $\Phi, f_{l}(\omega, x) \leq f_{m n}(\omega, x)+f_{l-m n}\left(T^{m n}(\omega, x)\right)$ and $f_{l-m n}\left(T^{m n}(\omega, x)\right) \leq$ $\sum_{i=m n}^{l-1} f_{1}\left(T^{i}(\omega, x)\right)$. Then, for $k \geq l$,

$$
\begin{aligned}
& P_{\text {pre, }, l, \omega}\left(T, \Phi, \epsilon,\left(T_{\omega}^{k}\right)^{-1} x\right) \\
& =\sup \left\{\sum_{y \in E} \exp f_{l}(\omega, y): E \text { is an }(\omega, l, \epsilon) \text {-separated set for } T \text { on }\left(T_{\omega}^{k}\right)^{-1} x\right\} \\
& =\sup \left\{\sum_{y \in E} \exp \left(f_{m n}(\omega, y)+\sum_{j=m n}^{l-1} f_{1}\left(\vartheta^{j} \omega, T_{\omega}^{j} y\right)\right):\right. \\
& \left.E \text { is an }(\omega, l, \epsilon) \text {-separated set for } T \text { on }\left(T_{\omega}^{k}\right)^{-1} x\right\} \\
& \leq \exp \sum_{j=m n}^{l-1}\left\|f_{1}\left(\vartheta^{j} \omega\right)\right\|_{\infty} \sup \left\{\sum_{y \in E} \exp f_{m n}(\omega, y):\right. \\
& \left.E \text { is an }(\omega, n, \delta) \text {-separated set for } T^{m} \text { on }\left(T_{\omega}^{k}\right)^{-1} x\right\}
\end{aligned}
$$

Since $f_{1} \in \mathbf{L}_{\mathcal{E}}^{1}(\Omega, \mathcal{C}(X))$, so $\int \exp \sum_{j=m n}^{l-1}\left\|f_{1}\left(\vartheta^{j} \omega\right)\right\|_{\infty} \mathrm{d} \mathbf{P}(\omega)<\infty$. Let $k=k_{1} m+q, k_{1}, q \in \mathbb{N}$, so $k_{1} \geq n$, then by the definition of $P_{\text {pre }}(T, \Phi, \epsilon)$, it is not hard to see that

$$
m P_{\text {pre }}(T, \Phi, \epsilon) \leq P_{\text {pre }}\left(T^{m}, \Phi^{m}, \delta\right) .
$$

If $\epsilon \rightarrow 0$, then $\delta \rightarrow 0$. Hence, we obtain $m P_{\text {pre }}(T, \Phi) \leq P_{\text {pre }}\left(T^{m}, \Phi^{m}\right)$ and complete the proof.

\section{Subadditive Pre-Image Variational Principle}

In this section, we are concerned with the variational principle for the subadditive pre-image topological pressure.

We first give a variational inequality among the subadditive pre-image topological pressure, the pre-image measure-theoretic entropy and the subadditive potential with respect to the invariant measures.

Theorem 1. Let $T$ be a continuous bundle RDS on $\mathcal{E}$ and $\Phi=\left\{f_{n}\right\}_{n=1}^{\infty}$ be subadditive. Then, for each $\mu \in \mathcal{M}_{\mathbf{P}}^{1}(\mathcal{E}, T)$ with $\Phi^{*}(\mu)>-\infty$,

$$
h_{\text {pre }, \mu}^{(r)}(T)+\Phi^{*}(\mu) \leq P_{\text {pre }}(T, \Phi) .
$$

Proof. Let $\mu \in \mathcal{M}_{\mathbf{P}}^{1}(\mathcal{E}, T), \Phi^{*}(\mu)>-\infty, \mathcal{P}=\left\{P_{1}, \cdots, P_{k}\right\}$, be a finite measurable partition of $X$, and $\epsilon$ be a positive number with $\epsilon k \log k<1$. Denote by $\left.\mathcal{P}(\omega)=\left\{P_{1}(\omega), \cdots, P_{k}(\omega)\right\}\right\}, P_{i}(\omega)=$ $P_{i} \cap \mathcal{E}_{\omega}, i=1, \cdots, k$, the corresponding partition of $\mathcal{E}_{\omega}$. By the regularity of $\mu$, we can find compact sets $Q_{i} \subset P_{i}, 1 \leq i \leq k$, such that

$$
\int \mu_{\omega}\left(P_{i}(\omega) \backslash Q_{i}(\omega)\right) \mathrm{d} \mathbf{P}(\omega)<\epsilon,
$$

where $Q_{i}(\omega)=Q_{i} \cap \mathcal{E}_{\omega}$. Let $\mathcal{Q}(\omega)=\left\{Q_{0}(\omega), \cdots, Q_{k}(\omega)\right\}$ be the partition of $\mathcal{E}_{\omega}$, where $Q_{0}(\omega)=$ $\mathcal{E}_{\omega} \backslash \bigcup_{i=1}^{k} Q_{i}(\omega)$. Then, by the proof of Theorem 4 of Zhu [30] and Lemma II. 1.3 of Kifer [37], the following inequality holds (see [30] for details),

$$
h_{\text {pre }, \mu}^{(r)}(T, \Omega \times \mathcal{P}) \leq h_{\text {pre }, \mu}^{(r)}(T, \Omega \times \mathcal{Q})+1,
$$

where $\Omega \times \mathcal{P}$ (respectively by $\Omega \times \mathcal{Q}$ ) denotes the partition of $\Omega \times X$ into sets $\Omega \times P_{i}$ (respectively by $\left.\Omega \times Q_{i}\right)$. 
For each $k \geq 0$, denote $A_{\mathcal{E}_{\vartheta^{k}}}$ be the partition of $\mathcal{E}_{\vartheta^{k} \omega}$ into points, and $\mathcal{A}_{\mathcal{E}_{\vartheta^{k}}}$ be the $\sigma$-algebra generated by $A_{\mathcal{E}_{\theta^{k} \omega}}$. Obviously, $\mathcal{A}_{\mathcal{E}_{\theta^{k} \omega}} \subset \mathcal{B}_{\mathcal{E}_{\theta^{k} \omega}}$ and so $\left(T_{\omega}^{k}\right)^{-1} \mathcal{A}_{\mathcal{E}_{\theta^{k}}} \subset\left(T_{\omega}^{k}\right)^{-1} \mathcal{B}_{\mathcal{E}_{\theta^{k} \omega}}$. Set

$$
\mathcal{Q}_{n}(\omega)=\bigvee_{i=0}^{n-1}\left(T_{\omega}^{i}\right)^{-1} \mathcal{Q}\left(\vartheta^{i} \omega\right),
$$

then $H_{\mu_{\omega}}\left(\mathcal{Q}_{n}(\omega) \mid\left(T_{\omega}^{k}\right)^{-1} \mathcal{B}_{\mathcal{E}_{\vartheta k}}\right) \leq H_{\mu_{\omega}}\left(\mathcal{Q}_{n}(\omega) \mid\left(T_{\omega}^{k}\right)^{-1} \mathcal{A}_{\mathcal{E}_{\vartheta k} \omega}\right)$.

Let $\mu_{\omega}=\int_{\mathcal{E}_{g^{k}}} \mu_{\omega, y} \mathrm{~d} \mu_{\omega} \circ\left(T_{\omega}^{k}\right)^{-1} y$ be the decomposition of $\mu_{\omega}$ relative to the partition $\zeta=$ $\left(T_{\omega}^{k}\right)^{-1} A_{\mathcal{E}_{\vartheta^{k}}}$. By the continuity of $T$, we can choose the measures $\mu_{\omega, y}$ so that $\mu_{\omega, y}\left(\left(T_{\omega}^{k}\right)^{-1} y\right)=1$ for each $y$ (See [41]). Therefore,

$$
\begin{aligned}
& H_{\mu_{\omega}}\left(\mathcal{Q}_{n}(\omega) \mid\left(T_{\omega}^{k}\right)^{-1} \mathcal{B}_{\mathcal{E}_{\vartheta k} \omega}\right)+\int_{\mathcal{E}_{\omega}} f_{n}(\omega) d \mu_{\omega} \\
\leq & H_{\mu_{\omega}}\left(\mathcal{Q}_{n}(\omega) \mid\left(T_{\omega}^{k}\right)^{-1} \mathcal{A}_{\mathcal{E}_{\vartheta^{k} \omega}}\right)+\int_{\mathcal{E}_{\omega}} f_{n}(\omega) d \mu_{\omega} \\
= & \int_{\mathcal{E}_{\vartheta k} \omega}\left(H_{\mu_{\omega, y}}\left(\mathcal{Q}(\omega) \mid\left(T_{\omega}^{k}\right)^{-1} y\right)+\int_{\mathcal{E}_{\omega}} f_{n}(\omega) d \mu_{\omega, y}\right) d \mu_{\omega} \circ\left(T_{\omega}^{k}\right)^{-1} y .
\end{aligned}
$$

For each $C \in \mathcal{Q}_{n}(\omega)$ and $y \in \mathcal{E}_{\vartheta^{k} \omega}$ with $C \cap\left(T_{\omega}^{k}\right)^{-1} y \neq \varnothing$, denote

$$
f_{n}^{*}(\omega, C, y)=\sup \left\{f_{n}(\omega, x): x \in C \cap\left(T_{\omega}^{k}\right)^{-1} y\right\},
$$

and let $x(C)$ be the point in $\overline{C \cap\left(T_{\omega}^{k}\right)^{-1} y}$ such that $f_{n}(\omega, x(C))=f_{n}^{*}(\omega, C, y)$. Then, by the well-known inequality (See [42])

$$
\sum_{1 \leq i \leq m} p_{i}\left(a_{i}-\log p_{i}\right) \leq \sum_{1 \leq i \leq m} \exp a_{i}
$$

where each $a_{i}$ is a real number, $p_{i} \geq 0$ and $\sum_{i=1}^{k} p_{i}=1$, we have

$$
\begin{aligned}
& H_{\mu_{\omega, y}}\left(\mathcal{Q}_{n}(\omega)\right)+\int_{\mathcal{E}_{\omega}} f_{n}(\omega) d \mu_{\omega, y} \\
\leq & \sum_{\substack{C \in \mathcal{Q}(\omega) \\
C \cap\left(T_{\omega}^{k}\right)^{-1} y \neq \varnothing}} \mu_{\omega, y}(C)\left(-\log \mu_{\omega, y}(C)+f_{n}^{*}(\omega, C, y)\right) \\
\leq & \log \sum_{\substack{C \in \mathcal{Q}(\omega) \\
C \cap\left(T_{\omega}^{k}\right)^{-1} y \neq \varnothing}} \exp \left(f_{n}^{*}(\omega, C, y)\right)
\end{aligned}
$$

Let $\mathcal{R}=\left\{Q_{0} \cup Q_{1}, \cdots, Q_{0} \cup Q_{k}\right\}$ be the open cover set of $X$, and $\delta$ be the Lebesgue number for $\mathcal{R}$. Then, for every $\omega, \mathcal{R}_{\omega}=\left\{Q_{0}(\omega) \cup Q_{1}(\omega), \cdots, Q_{0}(\omega) \cup Q_{k}(\omega)\right\}$ is the open cover of $\mathcal{E}_{\omega}$ and $\delta$ is a Lebesgue number of $\mathcal{R}_{\omega}$. If $D$ is another element of $\mathcal{Q}_{n}(\omega)$ with $D \cap\left(T_{\omega}^{k}\right)^{-1} y \neq \varnothing$ such that $d(x(C), x(D))<\delta$, then $x(C)$ and $x(D)$ are in the same element of $\mathcal{R}_{\omega}$, say $Q_{0}(\omega) \cup Q_{j}(\omega), 0 \leq j<$ $k+1$. Hence, for each $C$, there are at most $2^{n}$ elements $D$ of $\mathcal{Q}_{n}(\omega)$ such that

$$
d_{n}^{\omega}(x(C), x(D))=\max _{0 \leq j<n}\left\{d\left(T_{\omega}^{j}(x(C)), T_{\omega}^{j}(x(D))\right\}<\delta .\right.
$$

Now, an $(\omega, n, \delta)$-separated set $E_{\omega, y}$ can be constructed, which satisfies

$$
\sum_{\substack{C \in \mathcal{Q}(\omega) \\ C \cap\left(T_{\omega}^{k}\right)^{-1} y \neq \varnothing}} f_{n}^{*}(\omega, C, y) \leq 2^{n} \sum_{z \in E_{\omega, y}} f_{n}(\omega, z) .
$$


For this purpose, we first select the point $x\left(C_{1}\right)$ such that

$$
f_{n}^{*}\left(\omega, C_{1}, y\right)=\max _{\substack{C \in \mathcal{Q}(\omega) \\ C \cap\left(T_{\omega}^{k}\right)^{-1} y \neq \varnothing}} f_{n}^{*}(\omega, C, y),
$$

then select the second point $x\left(C_{2}\right)$ such that

$$
f_{n}^{*}\left(\omega, C_{2}, y\right)=\max _{\substack{C^{\prime} \in \mathcal{Q}_{n}(\omega) \\ C^{\prime} \cap\left(T_{\omega}^{k}\right)^{-1} y \neq \varnothing \\ d_{n}^{\omega}\left(x\left(C_{1}\right), x\left(C^{\prime}\right)\right) \geq \delta}} f_{n}^{*}\left(\omega, C^{\prime}, y\right),
$$

the third point $x\left(C_{3}\right)$ such that

$$
f_{n}^{*}\left(\omega, C_{3}, y\right)=\max _{\substack{C^{\prime \prime} \in \mathcal{Q}_{n}(\omega) \\ C^{\prime \prime} \cap\left(T_{\omega}^{k}\right)^{-1} y \neq \varnothing \\ d_{n}^{\omega}\left(x\left(C_{1}\right), x\left(C^{\prime \prime}\right)\right) \geq \delta \\ d_{n}^{\omega}\left(x\left(C_{2}\right), x\left(C^{\prime \prime}\right)\right) \geq \delta}} f_{n}^{*}\left(\omega, C^{\prime \prime}, y\right)
$$

continue this process, a finite step $m$ can complete this selection since $\mathcal{Q}_{n}(\omega)$ is finite. Let $E_{\omega, y}=$ $\left\{x\left(C_{1}\right), \cdots, x\left(C_{m}\right)\right\}$. Obviously $E_{\omega, y}$ is an $(\omega, n, \delta)$-separated set. By the above analysis, for each step, we delete at most $2^{n}$ elements of $\mathcal{Q}_{n}(\omega)$, so the inequality (10) holds.

Using the inequality (8), (9) and (10), we have

$$
\begin{aligned}
& H_{\mu_{\omega}}\left(\mathcal{Q}_{n}(\omega) \mid\left(T_{\omega}^{k}\right)^{-1} \mathcal{B}_{\mathcal{E}_{\vartheta k} \omega}\right)+\int_{\mathcal{E}_{\omega}} f_{n}(\omega) d \mu_{\omega} \\
\leq & \int_{\mathcal{E}_{\vartheta \vartheta^{k}}}\left(n \log 2+\log \sum_{z \in E_{\omega, y}} f_{n}(\omega, z)\right) d \mu_{\omega} \circ\left(T_{\omega}^{k}\right)^{-1} y \\
\leq & n \log 2+\int_{\mathcal{E}_{\vartheta k}} \log P_{\text {pre }, n, \omega}\left(T, \Phi, \delta,\left(T_{\omega}^{k}\right)^{-1} y\right) d \mu_{\omega} \circ\left(T_{\omega}^{k}\right)^{-1} y \\
\leq & n \log 2+\sup _{y \in \mathcal{E}_{\vartheta k}} \log P_{\text {pre }, n, \omega}\left(T, \Phi, \delta,\left(T_{\omega}^{k}\right)^{-1} y\right) \\
\leq & n \log 2+\sup _{x \in X_{\mathcal{E}}} \log P_{\text {pre }, n, \omega}\left(T, \Phi, \delta,\left(T_{\omega}^{k}\right)^{-1} x\right)
\end{aligned}
$$

By our assumed integrable condition, integrating this inequality against $\mathbf{P}$ and letting $k \rightarrow \infty$, dividing by $n$ and passing to $\lim \sup _{n \rightarrow \infty}$, by Proposition 1, the equality (6) and $\Phi^{*}(\mu)>-\infty$, we get

$$
h_{\mathrm{pre}, \mu}^{(r)}(T, \Omega \times \mathcal{Q})+\Phi^{*}(\mu) \leq \log 2+P_{\text {pre }}(T, \Phi, \delta)
$$

Hence, following from the inequality (7),

$$
\begin{gathered}
h_{\text {pre }, \mu}^{(r)}(T, \Omega \times \mathcal{P})+\Phi^{*}(\mu) \\
\leq 1+h_{\text {pre }, \mu}^{(r)}(T, \Omega \times \mathcal{Q})+\Phi^{*}(\mu) \leq 1+\log 2+P_{\text {pre }}(T, \Phi, \delta)
\end{gathered}
$$

By the arbitrariness of $\mathcal{P}$ and $\delta$, it follows that

$$
h_{\mathrm{pre}, \mu}^{(r)}(T)+\Phi^{*}(\mu) \leq 1+\log 2+P_{\mathrm{pre}}(T, \Phi)
$$

Since $h_{\text {pre, } \mu}^{(r)}\left(T^{n}\right)=n h_{\text {pre, } \mu}^{(r)}(T)$ (see Proposition 4 in [30]), the same arguments as above applied to $T^{n}$ in place of $T$; then, by $\left(\Phi^{n}\right)^{*}(\mu)=n \Phi^{*}(\mu)$, we obtain

$$
n\left(h_{\mathrm{pre}, \mu}^{(r)}(T)+\Phi^{*}(\mu)\right) \leq 1+\log 2+P_{\text {pre }}\left(T^{n}, \Phi^{n}\right) .
$$


Using Lemma 2, dividing by $n$ and letting $n \rightarrow \infty$, we conclude that

$$
h_{\text {pre }, \mu}^{(r)}(T)+\Phi^{*}(\mu) \leq P_{\text {pre }}(T, \Phi) .
$$

The following lemma is necessary in the proof of Proposition 2. It is a generalization of Cao's result [11] for the deterministic case and is proved in [34] as follows.

Lemma 3. For a sequence probability measures $\left\{\mu_{n}\right\}_{n=1}^{\infty}$ in $\mathcal{P}_{\mathbf{P}}(\mathcal{E})$, where $\mu_{n}=\frac{1}{n} \sum_{i=0}^{n-1} \Theta^{i} v_{n}$ and $\left\{v_{n}\right\}_{n=1}^{\infty} \subset$ $\mathcal{P}_{\mathbf{P}}(\mathcal{E})$, if $\left\{n_{i}\right\}$ is some subsequence of natural numbers $\mathbb{N}$ such that $\mu_{n_{i}} \rightarrow \mu \in \mathcal{M}_{\mathbf{P}}^{1}(\mathcal{E}, T)$; then, for any $k \in \mathbb{N}$,

$$
\limsup _{i \rightarrow \infty} \frac{1}{n_{i}} \int f_{n_{i}}(\omega, x) \mathrm{d} v_{n_{i}} \leq \frac{1}{k} \int f_{k} \mathrm{~d} \mu
$$

In particular, the left part is no more than $\Phi^{*}(\mu)$.

Proof. For $0 \leq j<k$ and $n \geq 2 k$, by the subadditivity of $\Phi$,

$$
\begin{aligned}
f_{n} & \leq f_{n-j} \Theta^{j}+f_{j} \\
& \leq\left(f_{k}+f_{k} \Theta^{k}+\cdots+f_{k} \Theta^{\left[\frac{n-j}{k}-1\right] k}+f_{n-j-\left[\frac{n-j}{k}\right] k} \Theta^{\left[\frac{n-j}{k}\right] k}\right) \Theta^{j}+f_{j} \\
& =\sum_{l=0}^{\left[\frac{n-j}{k}-1\right]} f_{k} \Theta^{l k+j}+\left(f_{n-j-\left[\frac{n-j}{k}\right] k} \Theta^{\left[\frac{n-j}{k}\right] k+j}+f_{j}\right),
\end{aligned}
$$

where $[a]$ denotes the integer part of $a$.

Summing over $j$ and dividing by $k$,

$$
\begin{aligned}
f_{n} & \leq \frac{1}{k} \sum_{j=0}^{k-1} \sum_{l=0}^{\left[\frac{n-j}{k}-1\right]} f_{k} \Theta^{l k+j}+\frac{1}{k} \sum_{j=0}^{k-1}\left(f_{n-j-\left[\frac{n-j}{k}\right] k} \Theta^{\left[\frac{n-j}{k}\right] k+j}+f_{j}\right) \\
& =\frac{1}{k} \sum_{s=0}^{n-k} f_{k} \Theta^{s}+\frac{1}{k} \sum_{j=0}^{k-1}\left(f_{n-j-\left[\frac{n-j}{k}\right] k} \Theta^{\left[\frac{n-j}{k}\right] k+j}+f_{j}\right) .
\end{aligned}
$$

Since $n-j-\left[\frac{n-j}{k}\right] \leq k$ and $f_{r} \leq \sum_{t=0}^{r-1} f_{1} \Theta^{t}$, where $1 \leq r \leq k$, integrating this inequality, then by $f_{1} \in \mathbf{L}_{\mathcal{E}}^{1}(\Omega, \mathcal{C}(X))$,

$$
\begin{aligned}
\int f_{n} d v_{n} & \leq \frac{1}{k} \int \sum_{s=0}^{n-k} f_{k} \Theta^{s} d v_{n}+\frac{1}{k} \int \sum_{j=0}^{k-1}\left(f_{n-j-\left[\frac{n-j}{k}\right] k} \Theta^{\left[\frac{n-j}{k}\right] k+j}+f_{j}\right) d v_{n} \\
& \leq \frac{1}{k} \int \sum_{s=0}^{n-k} f_{k} \Theta^{s} d v_{n}+\frac{1}{k} 2 k^{2} \int\|f(\omega)\|_{\infty} d \mathbf{P}(\omega) \\
& =\frac{n-k+1}{k} \int f_{k} d \mu_{n}^{\prime}+2 k \int\|f(\omega)\|_{\infty} d \mathbf{P}(\omega)
\end{aligned}
$$

where $\mu_{n}^{\prime}=\frac{1}{n-k+1} \sum_{s=0}^{n-k} \Theta^{s} v_{n}$, since, for any $f \in \mathbf{L}_{\mathcal{E}}^{1}(\Omega, \mathcal{C}(X))$,

$$
\begin{aligned}
& n \int f d \mu_{n}-(n-k+1) \int f d \mu_{n}^{\prime} \\
= & \sum_{i=n-k+1}^{n-1} \int f \Theta^{i} d v_{n} \leq k \int\|f(\omega)\|_{\infty} d \mathbf{P}(\omega) .
\end{aligned}
$$


Dividing by $n$ and letting $n \rightarrow \infty$, we get

$$
\lim _{n \rightarrow \infty} \int f \mathrm{~d} \mu_{n}=\lim _{n \rightarrow \infty} \int f \mathrm{~d} \mu_{n}^{\prime} .
$$

Observing that $\lim _{i \rightarrow \infty} \mu_{n_{i}}^{\prime}=\mu$, which follows from $\left\{\mu_{n_{i}}\right\} \rightarrow \mu$, we have

$$
\lim _{i \rightarrow \infty} \int f_{k} \mathrm{~d} \mu_{n_{i}}^{\prime}=\int f_{k} \mathrm{~d} \mu
$$

Replacing $n$ by $n_{i}$ in Equation (12), dividing by $n_{i}$ and passing to $\lim \sup _{i \rightarrow \infty}$, then Equation (16) follows by Equation (13). Letting $k \rightarrow \infty$, then the result holds.

For the subadditive sequences of potentials, by constructing a maximal invariant measure, one can set up a relationship between the subadditive pre-image topological pressure and the pre-image measure-theoretic entropy on continuous bundle random dynamical systems.

Proposition 2. Let $T$ be a continuous bundle RDS on $\mathcal{E}$ and $\Phi=\left\{f_{n}\right\}_{n=1}^{\infty}$ be subadditive. If $P_{\text {pre }}(T, \Phi)>$ $-\infty$, there exists some $\mu \in \mathcal{M}_{\mathbf{P}}^{1}(\mathcal{E}, T)$ with $\Phi^{*}(\mu)>-\infty$ satisfying

$$
P_{\text {pre }}(T, \Phi) \leq h_{\text {pre }, \mu}^{(r)}(T)+\Phi^{*}(\mu) .
$$

Proof. Choose some small positive nonrandom $\epsilon>0$ with $P_{\text {pre }}(T, \Phi, \epsilon)>-\infty$. By Lemma 1, we can also choose a positive integer sequence $n_{i} \rightarrow \infty, k_{i} \geq n_{i}$, and points $x_{\vartheta k_{i} \omega} \in \mathcal{E}_{\vartheta k_{i} \omega}$ for each $\omega \in \Omega$ such that

$$
P_{\text {pre }}(T, \Phi, \epsilon)=\lim _{i \rightarrow \infty} \frac{1}{n_{i}} \int \log P_{\text {pre }, n_{i}, \omega}\left(T, \Phi, \epsilon,\left(T_{\omega}^{k_{i}}\right)^{-1} x_{\vartheta^{k_{i}}}\right) d \mathbf{P}(\omega) .
$$

Fix $n_{i}$ and employ Lemma 1 in order to choose a measurable in $\omega$ family of maximal $\left(\omega, n_{i}, \epsilon\right)$-separated sets $G\left(\omega, n_{i}, \epsilon\right) \subset\left(T_{\omega}^{k_{i}}\right)^{-1} x_{\vartheta \vartheta_{i} \omega} \subset \mathcal{E}_{\omega}$ such that

$$
\sum_{x \in G\left(\omega, n_{i} \epsilon\right)} \exp f_{n_{i}}(\omega, x) \geq \frac{1}{e} P_{\text {pre, } n_{i}, \omega}\left(T, \Phi, \epsilon,\left(T_{\omega}^{k_{i}}\right)^{-1} x_{\vartheta^{k_{i}} \omega}\right)
$$

Next, define probability measures $v^{(i)}$ on $\mathcal{E}$ via their measurable disintegrations

$$
v_{\omega}^{(i)}=\frac{\sum_{x \in G\left(\omega, n_{i} \epsilon\right)} \exp f_{n_{i}}(\omega, x) \delta_{x}}{\sum_{x \in G\left(\omega, n_{j} \epsilon\right)} \exp f_{n_{i}}(\omega, x)}
$$

so that $d v^{(i)}(\omega, x)=d v_{\omega}^{(i)}(x) d \mathbf{P}(\omega)$, and set

$$
\mu^{(i)}=\frac{1}{n_{i}} \sum_{j=0}^{n_{i}-1} \Theta^{j} v^{(i)}
$$

Then, by Lemma 2.1 (i)-(ii) in [15], we can choose a subsequence $n_{i_{l}}$ such that $\lim _{l \rightarrow \infty} \mu^{\left(i_{l}\right)}=\mu \in$ $\mathcal{M}_{\mathbf{P}}^{1}(\mathcal{E}, T)$. Without losing the generality, we still assume that $\lim _{i \rightarrow \infty} \mu^{(i)}=\mu$.

Next, choose a partition $\mathcal{P}=\left\{P_{1}, \cdots, P_{k}\right\}$ of $X$ with $\operatorname{diam} \mathcal{P} \leq \epsilon$ and such that and $\int \mu_{\omega}\left(\partial P_{i}\right) d \mathbf{P}(\omega)=0$ for all $1 \leq i \leq k$, where $\partial$ denotes the boundary. Set $\mathcal{P}(\omega)=\left\{P_{1}(\omega), \cdots, P_{k}(\omega)\right\}$, 
$P_{i}(\omega)=P_{i} \cap \mathcal{E}_{\omega}$. Since each element of $\bigvee_{l=0}^{n_{i}-1}\left(T_{\omega}^{l}\right)^{-1} \mathcal{P}\left(\vartheta^{l} \omega\right)$ contains at most one element of $G\left(\omega, n_{i}, \epsilon\right)$, by the inequality (14), we have

$$
\begin{aligned}
& H_{v_{\omega}^{(i)}}\left(\left.\bigvee_{l=0}^{n_{i}-1}\left(T_{\omega}^{l}\right)^{-1} \mathcal{P}\left(\vartheta^{l} \omega\right)\right|_{\left(T_{\omega}^{k_{i}}\right)^{-1} x_{\vartheta^{k_{i}}}}\right)+\int f_{n_{i}}(\omega) d v_{\omega}^{(i)} \\
= & \sum_{x \in G\left(\omega, n_{i} \epsilon\right)} v_{\omega}^{(i)}(\{x\})\left(-\log v_{\omega}^{(i)}(\{x\})+f_{n_{i}}(\omega, x)\right) \\
= & \log \sum_{x \in G\left(\omega, n_{i} \epsilon\right)} \exp f_{n_{i}}(\omega, x) \\
\geq & \log P_{\text {pre }, n_{i}, \omega}\left(T, f, \epsilon,\left(T_{\omega}^{k_{i}}\right)^{-1} x_{\vartheta^{k_{i}} \omega}\right)-1 .
\end{aligned}
$$

For $\omega \in \Omega$, let $\mathcal{C}_{\omega}$ denotes the subcollection of $\mathcal{B}_{\omega}^{-}$consisting of $\mu_{\omega}$-null sets. For any $\sigma$-algebra $\mathcal{A}$ of subsets of $\mathcal{E}_{\omega}$, there is an enlarged $\sigma$-algebra $\mathcal{A}_{\mathcal{C}_{\omega}}$ defined by $A \in \mathcal{A}_{\mathcal{C}_{\omega}}$ if and only if there are sets $B, M, N$ such that $A=B \cup M, B \in \mathcal{A}, N \in \mathcal{C}_{\omega}$ and $M \subset N$. The $\sigma$-algebra $\mathcal{B}_{\mathcal{C}_{\omega}}^{-}$is simply the standard $\mu_{\omega}$-completion of $\mathcal{B}_{\omega}^{-}$. Denote $\sigma$-algebra $\mathcal{B}_{\omega}^{k}=\left(\left(T_{\omega}^{k}\right)^{-1} \mathcal{B}_{\vartheta^{k}}\right)_{\mathcal{C}_{\omega}}$ for all $k \geq 1$. Since $T_{\omega}^{-1} \mathcal{C}_{\omega} \subset \mathcal{C}_{\omega}$ for each $\omega$, we have that $\mathcal{B}_{\omega}^{1} \supset \mathcal{B}_{\omega}^{2} \supset \cdots$. Let $\mathcal{B}_{\omega}^{\infty}=\bigcap_{k \geq 1} \mathcal{B}_{\omega}^{k}$, then $\mathcal{B}_{\omega}^{-} \subset \mathcal{B}_{\mathcal{C}_{\omega}}^{-} \subset \mathcal{B}_{\omega}^{\infty}$ and $\left(T_{\omega}^{l}\right)^{-1} \mathcal{B}_{\vartheta^{l} \omega}^{k} \subset \mathcal{B}_{\omega}^{l+k}$ for all $l \geq 1$.

Similarly, let $\mathcal{C}_{\mathcal{E}}$ denote the subcollection of $(\mathcal{F} \times \mathcal{B})_{\mathcal{E}}^{-}$consisting of $\mu$-null sets. Define $(\mathcal{F} \times$ $\mathcal{B})_{\mathcal{E}}^{k}=\left(\Theta^{-k}(\mathcal{F} \times \mathcal{B})_{\mathcal{E}}\right)_{\mathcal{C}_{\mathcal{E}}}$ and $(\mathcal{F} \times \mathcal{B})_{\mathcal{E}}^{\infty}=\bigcap_{k \geq 1}(\mathcal{F} \times \mathcal{B})_{\mathcal{E}}^{k}$. Clearly, $(\mathcal{F} \times \mathcal{B})_{\mathcal{E}}^{1} \supset(\mathcal{F} \times \mathcal{B})_{\mathcal{E}}^{2} \supset \cdots$, $(\mathcal{F} \times \mathcal{B})_{\mathcal{E}}^{-} \subset(\mathcal{F} \times \mathcal{B})_{\mathcal{E}}^{\infty} \subset(\mathcal{F} \times \mathcal{B})_{\mathcal{E}}^{k}$ and $\Theta^{-l}\left(\mathcal{F}_{\mathcal{E}} \vee(\mathcal{F} \times \mathcal{B})_{\mathcal{E}}^{k}\right) \subset \mathcal{F}_{\mathcal{E}} \vee(\mathcal{F} \times \mathcal{B})_{\mathcal{E}}^{l+k}$ for all $l \geq 1$. As a similar proof in Proposition 4, for any finite partition $\eta$ of $\Omega \times X$, we have

$$
H_{v^{(i)}}\left(\eta \mid \mathcal{F}_{\mathcal{E}} \vee(\mathcal{F} \times \mathcal{B})_{\mathcal{E}}^{k}\right)=\int H_{v_{\omega}^{(i)}}\left(\eta \mid \mathcal{B}_{\omega}^{k}\right) d \mathbf{P}(\omega)
$$

Note that $v_{\omega}^{(i)}$ is supported on $\left(T_{\omega}^{k_{i}}\right)^{-1} x_{\vartheta k^{k_{i},}}$, the canonical system of conditional measures induced by $v_{\omega}^{(i)}$ on the measurable partition $\left\{\left(T_{\omega}^{k_{i}}\right)^{-1} x \mid x \in \mathcal{E}_{\vartheta^{k_{i}} \omega}\right\}$ reduces to a single measure on the set $\left(T_{\omega}^{k_{i}}\right)^{-1} x_{\vartheta^{k_{i}} \omega^{\prime}}$ which we may identify with $v_{\omega}^{(i)}$. Moreover, since each $A \in \mathcal{B}_{\omega}^{k_{i}}$ can be expressed as the disjoint union $A=B \cup C$, where $B \in\left(T_{\omega}^{k_{i}}\right)^{-1} \mathcal{B}_{\vartheta^{k_{i}}}$ and $C \in \mathcal{C}_{\omega}$, since $v_{\omega}^{(i)}$ is supported on elements of $\left(T_{\omega}^{k_{i}}\right)^{-1} x_{\vartheta^{k_{i}} \omega^{\prime}}$, then $v_{\omega}^{(i)}(C)=0$. Hence, for any finite partition $\gamma$, we have

$$
H_{v_{\omega}^{(i)}}\left(\gamma \mid \mathcal{B}_{\omega}^{k_{i}}\right)=H_{v_{\omega}^{(i)}}\left(\left.\gamma\right|_{\left(T_{\omega}^{k_{i}}\right)^{-1} x_{\theta^{k_{i}}}}\right) .
$$

Let $\mathcal{Q}=\left\{Q_{1}, \cdots, Q_{k}\right\}$, where $Q_{i}=\left(\Omega \times P_{i}\right) \cap \mathcal{E}$, then $\mathcal{Q}$ is a partition of $\mathcal{E}$ and $Q_{i}(\omega)=\{x \in$ $\left.\mathcal{E}_{\omega}:(\omega, x) \in Q_{i}\right\}=P_{i}(\omega)$. Integrating in the Equation (15) against $\mathbf{P}$, then by Equations (16) and (17), we obtain the inequality

$$
\begin{aligned}
& H_{v^{(i)}}\left(\bigvee_{l=0}^{n_{i}-1}\left(\Theta^{l}\right)^{-1} \mathcal{Q} \mid \mathcal{F}_{\mathcal{E}} \vee(\mathcal{F} \times \mathcal{B})_{\mathcal{E}}^{k_{i}}\right)+\int f_{n_{i}} d v^{(i)} \\
& \geq \int \log P_{\text {pre }, n_{i}, \omega}\left(T, \Phi, \epsilon,\left(T_{\omega}^{k_{i}}\right)^{-1} x_{\vartheta^{k_{i}}}\right) d \mathbf{P}(\omega)-1
\end{aligned}
$$

Considering $q, n_{i} \in \mathbb{N}$ such that $1<q<n_{i}$ and let $a(s)$ denote the integer part of $\left(n_{i}-s\right) q^{-1}$ for all $0 \leq s<q$. Then, clearly, for each $s$,

$$
\bigvee_{l=0}^{n_{i}-1}\left(\Theta^{l}\right)^{-1} \mathcal{Q}=\bigvee_{r=0}^{a(s)-1}\left(\Theta^{r q+s}\right)^{-1} \bigvee_{t=0}^{q-1}\left(\Theta^{t}\right)^{-1} \mathcal{Q} \vee \bigvee_{l \in S}\left(\Theta^{l}\right)^{-1} \mathcal{Q}
$$

where card $S \leq 2 q$. 
Since $\operatorname{card} \mathcal{Q}=k$, taking into account the subadditivity of conditional entropy (see Section 2.1 in [37]), it follows that

$$
\begin{aligned}
& H_{v^{(i)}}\left(\bigvee_{l=0}^{n_{i}-1}\left(\Theta^{l}\right)^{-1} \mathcal{Q} \mid \mathcal{F}_{\mathcal{E}} \vee(\mathcal{F} \times \mathcal{B})_{\mathcal{E}}^{k_{i}}\right) \\
\leq & \sum_{r=0}^{a(s)-1} H_{v^{(i)}}\left(\left(\Theta^{r q+s}\right)^{-1} \bigvee_{t=0}^{q-1}\left(\Theta^{t}\right)^{-1} \mathcal{Q} \mid \mathcal{F}_{\mathcal{E}} \vee(\mathcal{F} \times \mathcal{B})_{\mathcal{E}}^{k_{i}}\right)+2 q \log k \\
\leq & \sum_{r=0}^{a(s)-1} H_{v^{(i)}}\left(\left(\Theta^{r q+s}\right)^{-1} \bigvee_{t=0}^{q-1}\left(\Theta^{t}\right)^{-1} \mathcal{Q} \mid\left(\Theta^{r q+s}\right)^{-1}\left(\mathcal{F}_{\mathcal{E}} \vee(\mathcal{F} \times \mathcal{B})_{\mathcal{E}}^{k_{i}}\right)\right)+2 q \log k \\
= & \sum_{r=0}^{a(s)-1} H_{\Theta^{r q+s_{v}} v^{(i)}}\left(\bigvee_{t=0}^{q-1}\left(\Theta^{t}\right)^{-1} \mathcal{Q} \mid \mathcal{F}_{\mathcal{E}} \vee(\mathcal{F} \times \mathcal{B})_{\mathcal{E}}^{k_{i}}\right)+2 q \log k
\end{aligned}
$$

Summing this inequality over $s \in\{0,1, \cdots, q-1\}$, we get

$$
\begin{aligned}
& q H_{v^{(i)}}\left(\bigvee_{l=0}^{n_{i}-1}\left(\Theta^{l}\right)^{-1} \mathcal{Q} \mid \mathcal{F}_{\mathcal{E}} \vee(\mathcal{F} \times \mathcal{B})_{\mathcal{E}}^{k_{i}}\right) \\
\leq & \sum_{m=0}^{n_{i}-1} H_{\Theta^{m} v^{(i)}} \mid\left(\bigvee_{t=0}^{q-1}\left(\Theta^{t}\right)^{-1} \mathcal{Q} \mid \mathcal{F}_{\mathcal{E}} \vee(\mathcal{F} \times \mathcal{B})_{\mathcal{E}}^{k_{i}}\right)+2 q^{2} \log k \\
\leq & n_{i} H_{\mu^{(i)}}\left(\bigvee_{t=0}^{q-1}\left(\Theta^{t}\right)^{-1} \mathcal{Q} \mid \mathcal{F}_{\mathcal{E}} \vee(\mathcal{F} \times \mathcal{B})_{\mathcal{E}}^{k_{i}}\right)+2 q^{2} \log k \\
\leq & n_{i} H_{\mu^{(i)}}\left(\bigvee_{t=0}^{q-1}\left(\Theta^{t}\right)^{-1} \mathcal{Q} \mid \mathcal{F}_{\mathcal{E}} \vee(\mathcal{F} \times \mathcal{B})_{\mathcal{E}}^{\infty}\right)+2 q^{2} \log k
\end{aligned}
$$

where the second inequality, as in Kifer's works [15], relies on the general property of conditional entropy of partition $\sum_{i} p_{i} H_{\eta_{i}}(\xi \mid \mathcal{A}) \leq H_{\sum_{i} p_{i} \eta_{i}}(\xi \mid \mathcal{A})$ which holds for any finite partition $\xi$, $\sigma$-algebra $\mathcal{A}$, probability measures $\eta_{i}$, and probability vector $\left(p_{i}\right), i=1, \ldots, n$, in view of the convexity of $t \log t$ in the same way as in the unconditional case (cf. [42] (pp.183 and 188)). This together with the Equation (18) yields

$$
\begin{aligned}
& q \int \log P_{\text {pre }, n_{i}, \omega}\left(T, \Phi, \epsilon,\left(T_{\omega}^{k_{i}}\right)^{-1} x_{\vartheta^{k_{i}} \omega}\right) d \mathbf{P}(\omega)-q \\
\leq & n_{i} H_{\mu^{(i)}}\left(\bigvee_{t=0}^{q-1}\left(\Theta^{t}\right)^{-1} \mathcal{Q} \mid \mathcal{F}_{\mathcal{E}} \vee(\mathcal{F} \times \mathcal{B})_{\mathcal{E}}^{\infty}\right)+2 q^{2} \log k+q \int f_{n_{i}} d v^{(i)} .
\end{aligned}
$$

Diving by $n_{i}$, passing to the lim sup $\operatorname{sum}_{i \rightarrow \infty}$, taking into account Lemma 3 and using the inequality 10 in [30] in the case of the measurable set $\mathcal{E}$, i.e.,

$$
\begin{gathered}
H_{\mu}\left(\bigvee_{t=0}^{q-1}\left(\Theta^{t}\right)^{-1} \mathcal{Q} \mid \mathcal{F}_{\mathcal{E}} \vee(\mathcal{F} \times \mathcal{B})_{\mathcal{E}}^{-}\right) \\
\geq \limsup _{n \rightarrow \infty} H_{\mu^{(i)}}\left(\bigvee_{t=0}^{q-1}\left(\Theta^{t}\right)^{-1} \mathcal{Q} \mid \mathcal{F}_{\mathcal{E}} \vee(\mathcal{F} \times \mathcal{B})_{\mathcal{E}}^{\infty}\right),
\end{gathered}
$$


we get that

$$
q P_{\text {pre }}(T, \Phi, \epsilon) \leq H_{\mu}\left(\bigvee_{t=0}^{q-1}\left(\Theta^{t}\right)^{-1} \mathcal{Q} \mid \mathcal{F}_{\mathcal{E}} \vee(\mathcal{F} \times \mathcal{B})_{\mathcal{E}}^{-}\right)+q \Phi^{*}(\mu),
$$

then $\Phi^{*}(\mu)>-\infty$ since $P_{\text {pre }}(T, \Phi)>-\infty$. Dividing by $q$ and letting $q \rightarrow \infty$, we have

$$
P_{\text {pre }}(T, \Phi, \epsilon) \leq h_{\text {pre }, \mu}^{(r)}(T, \mathcal{Q})+\Phi^{*}(\mu) \leq h_{\text {pre }, \mu}^{(r)}(T)+\Phi^{*}(\mu) .
$$

Letting $\epsilon \rightarrow 0$, we have $P_{\text {pre }}(T, \Phi) \leq h_{\text {pre, } \mu}^{(r)}(T)+\Phi^{*}(\mu)$ and complete the proof of Proposition 2.

It follows directly from Theorem 1 and Proposition 2 that the desired subadditive pre-image variational principle holds.

Theorem 2. Let $T$ be a continuous bundle $R D S$ on $\mathcal{E}, \Phi=\left\{f_{n}\right\}_{n=1}^{\infty}$ be subadditive. If $P_{\text {pre }}(T, \Phi)>-\infty$, then

$$
P_{\text {pre }}(T, \Phi)=\sup \left\{h_{\text {pre }, \mu}^{(r)}(T)+\Phi^{*}(\mu): \mu \in \mathcal{M}_{\mathbf{P}}^{1}(\mathcal{E}, T) \text { and } \Phi^{*}(\mu)>-\infty\right\} .
$$

Remark 3. In fact, $P_{\text {pre }}(T, \Phi)=-\infty$ is equivalent to $\Phi^{*}(\mu)=-\infty$ for all invariant measure $\mu \in \mathcal{M}_{\mathbf{P}}^{1}(\mathcal{E}, T)$. Obviously, if $P_{\text {pre }}(T, \Phi) \geq \sup \left\{h_{\text {pre, } \mu}^{(r)}(T)+\Phi^{*}(\mu)\right\}$, then, by $\Phi^{*}(\mu)>-\infty, P_{\text {pre }}(T, \Phi)>-\infty$. Thus, the restricted condition in the above theorem is only used in the oppositive direction " $\leq$ ".

\section{Conclusions}

In this paper, we set up a variational principle for subadditive pre-image topological pressure for continuous bundle random dynamical systems. The topological pressure herein is proved under mild restriction to be the supremum of the sum of the pre-image measure-theoretic entropy and Lyapunov exponent of the subadditive potentials over the set of invariant measures in the continuous bundle random dynamical systems. For the whole product space as a measurable set and the zero potential, the variational principle reduces to the maximal entropy principle for the general random dynamical systems. Moreover, for the trivial base space with a single point adhering to the additive potentials, it is just the pre-image variational principle in the deterministic environment. The method we adopt is still in the framework of Misiurewicz's perfect proof of the classical variational principle. The result obtained generalizes the extant ones to a more general continuous bundle random dynamical systems and might be useful for the study of dimension estimates on non-conformal random dynamical systems.

Author Contributions: All authors have contributed in equal amount to the paper. All authors have read and agreed to the published version of the manuscript.

Funding: The research is supported by the National Natural Science Foundation of China (Grant No. 11471114) and Undergraduate Training Program for Innovation and Entrepreneurship (Grant No. X19189).

Acknowledgments: We would like to thank the references for their constructive comments and valuable suggestions.

Conflicts of Interest: The authors declare no conflict of interest.

\section{References}

1. Bogenschütz, T. Entropy, pressure, and a variational principle for random dynamical systems. Random Comput. Dyn. 1992, 1, 99-116.

2. Bowen, R. Equilibrium States and the Ergodic Theory of Anosov Diffemorphisms; Volume 470 of Lecture Notes in Mathematics; Springer: Berlin/Heidelberg, Germany, 1975.

3. Denker, M.; Kifer, Y.; Stadlbauer, M. Thermodynamic formalism for random countable Markov shifts. Discret. Contin. Dyn. Syst. 2008, 22, 131-164. [CrossRef] 
4. Gaspard, P. Chaos, Scattering and Statistical Mechanics; Cambridge Nonlinear Science Series, 9; Cambridge University Press: Cambridge, UK, 1998.

5. Khanin, K.; Kifer, Y. Thermodynamic formalism for random transformations and statistical mechanics. Am. Math. Soc. Transl. 1996, 1172, 107-140.

6. Kifer, Y. Thermodynamic formalism for random transformations revisited. Stoch. Dyn. 2008, 8, 77-102. [CrossRef]

7. Pesin, Y. Dimension Theory in Dynamical Systems. Contemporary Views and Applications; University of Chicago Press: Chicago, IL, USA; London, UK, 1997.

8. Ruelle, D. Thermodynamic Formalism; Addison-Wesley: Reading, MA, USA, 1978.

9. Ruelle, D. Statistical mechanics on a compact set with $z^{v}$ action satisfying expansivenenss and specification. Trans. Am. Math. Soc. 1973, 187, 237-251. [CrossRef]

10. Falconer, K.J. A subadditive thermodynamic formalism for mixing repellers. J. Phys. A 1988, 21, L737-L742. [CrossRef]

11. Cao, Y.; Feng, D.; Huang, W. The thermodynamic formalism for sub-additive potentials. Discrete Contin. Dyn. Syst. 2008, 20, 259-273. [CrossRef]

12. Ban, J.; Cao, Y.; Hu, H. The dimensions of non-conformal repeller and average conformal repeller. Trans. Am. Math. Soc. 2010, 362, 727-751. [CrossRef]

13. Barreira, L. Nonadditive thermodynamic formalism: Equilibrium and gibbs measures. Discrete Contin. Dyn. Syst. 2006, 16, 279-305 [CrossRef]

14. Ledrappier, F.; Walters, P. A relativised variational principle for continuous transformations. J. Lond. Math. Soc. 1977, 2, 568-576. [CrossRef]

15. Kifer, Y. On the topological pressure for random bundle transformations. Transl. Am. Math. Soc. 2001, 202, 197-214.

16. Gundlach, V.M.; Kifer, Y. Expansiveness, specification, and Equilibrium states for random bundle transformations. Discret. Contin. Dyn. Syst. 2000, 6, 89-120. [CrossRef]

17. Feng, D.; Shu, L. Multifractal analysis for disintegrations of Gibbs measures and conditional Birkhoff averages. Ergod. Theory Dyn. Syst. 2009, 29, 885-918. [CrossRef]

18. Cheng, W.-C. Two-point pre-image entropy. Discrete Contin. Dyn. Syst. 2007, 17, 107-119. [CrossRef]

19. Fiebig, D.; Fiebig, U.; Nitecki, Z. Entropy and preimage sets. Ergod. Theory Dyn. Syst. 2003, 23, $1785-1806$. [CrossRef]

20. Hurley, M. On the topological entropy of maps. Ergod. Theory Dyn. Syst. 1995, 15, 557-568. [CrossRef]

21. Langevin, R.; Walczak, P. Entropie d'une dynamique. Comptes Rendus l'Académie Sci. Série Math. 1991, 312, 141-144.

22. Langevin, R.; Prezytycki, F. Entropie de l'image inverse d'une application. Bull. Soc. Math. Fr. 1991, 120, 237-250. [CrossRef]

23. Nitecki, Z.; Prezytycki, F. Preimage entropy for mappings. Int. J. Bifurc. Chaos 1999, 9, 1815-1843. [CrossRef]

24. Yan, K.; Zeng, F. Variational principles of partial pre-image entropy and conditional pre-image entropy. Sci. Sin. Math. 2019, 49, 681-698.

25. Cheng, W.-C. Pre-image entropy for free semigroup actions. Chaos Solitons Fractals 2016, 91, $286-290$. [CrossRef]

26. Misiurewicz, M.; Rodrigues, A. Counting preimages. Ergod. Theory Dyn. Syst. 2018, 38, 1837-1856. [CrossRef]

27. Hou, B.; Wang, X. Entropy of a semigroup of maps from a set-valued view. B Iran. Math. Soc. 2017, 43, 1821-1835.

28. Cheng, W.-C.; Newhouse, S. Pre-image entropy. Ergod. Theory Dyn. Syst. 2005, 25, 1091-1113. [CrossRef]

29. Zeng, F.; Yan, K.; Zhang, G. Pre-image pressure and invariant measures, Ergod. Theory Dyn. Syst. 2007, 27, 1037-1052. [CrossRef]

30. Zhu, Y. Preimage entropy for random dynamical systems. Discret. Contin. Dyn. Syst. 2007, 18, 829-851. [CrossRef]

31. Zhu, Y. Preimage pressure for random transformations. Ergod. Theory Dyn. Syst. 2009, 29, 1669-1687. [CrossRef]

32. Cao, Y.; Pesin, Y.; Zhao, Y. Dimension estimates for non-conformal repellers and continuity of sub-additive topological pressure. Gem. Funct. Anal. 2019, 29, 1325-1368. [CrossRef] 
33. Misiurewicz, M. A short proof of the variational principle for a $\mathbb{Z}_{+}^{n}$ action on a compact space. Asterisque 1976, 40, 147-157.

34. Ma, X.; Chen, E. Variational principle for subadditive sequence of potentials in bundle random dynamical systems. Stoch. Dyn. 2009, 9, 205-215. [CrossRef]

35. Dudley, R.M. Real Analysis and Probability; Wadsworth and Brooks/Cole: Pacific Grove, CA, USA, 1989.

36. Arnold, L. Random Dynamical Systems; Springer: New York, NY, USA, 1998.

37. Kifer, Y. Ergodic Thoery of Random Transformations; Birkhäuser: Boston, MA, USA, 1986.

38. Bogenschütz, T. Equilibrium States for Random Dynamical Systems. Ph.D. Thesis, Universität Bremen, Bremen, Germany, 1993.

39. Ma, X.; Chen, E. Pre-image variational principle for bundle random dynamical systems. Discret. Contin. Dyn. Syst. 2009, 23, 957-972. [CrossRef]

40. Castaing, C.; Valadier, M. Convex Analysis and Measurable Multifunctions; Volume 580 of Lecture Notes in Mathematics; Springer: New York, NY, USA, 1977.

41. Bourbaki, N. Integration Ch. VI.; Springer: Berlin, Germany, 2004.

42. Walters, P. An Introduction to Ergodic Thoery; Springer: New York, NY, USA, 1982.

(C) 2020 by the authors. Licensee MDPI, Basel, Switzerland. This article is an open access article distributed under the terms and conditions of the Creative Commons Attribution (CC BY) license (http://creativecommons.org/licenses/by/4.0/). 